\title{
EXAMPLES OF CYCLIC POLYNOMIALLY BOUNDED OPERATORS THAT ARE NOT SIMILAR TO CONTRACTIONS, II
}

\author{
MARIA F. GAMAL'
}

\begin{abstract}
The question if polynomially bounded operator is similar to a contraction was posed by Halmos and was answered in the negative by Pisier. His counterexample is an operator of infinite multiplicity, while all its restrictions on invariant subspaces of finite multiplicity are similar to contractions. In [G16], cyclic polynomially bounded operators which are not similar to contractions was constructed. The construction was based on a perturbation of the sequence of finite dimensional operators which is uniformly polynomially bounded, but is not uniformly completely polynomially bounded, constructed by Pisier. In this paper, a cyclic polynomially bounded operator $T_{0}$ such that $T_{0}$ is not similar to a contraction and $\omega_{a}\left(T_{0}\right)=\mathbb{O}$, is constructed. Here $\omega_{a}(z)=\exp \left(a \frac{z+1}{z-1}\right)$, $z \in \mathbb{D}, a>0$, and $\mathbb{D}$ is the open unit disk. To obtain such $T_{0}$, a slight modification of the construction from [G16] is needed.
\end{abstract}

\section{INTRODUCTION}

Let $T$ be an operator on a Hilbert space. $T$ is called polynomially bounded, if there exists a constant $C$ such that

$$
\|p(T)\| \leq C\|p\|_{\infty} \text { for every polynomial } p,
$$

where $\|\cdot\|_{\infty}$ denotes the uniform norm on the open unit disk $\mathbb{D}$.

An operator $T$ is called a contraction if $\|T\| \leq 1$. Every contraction is polynomially bounded (von Neumann inequality, see, for example, [SFBK, Proposition I.8.3]).

The question if a polynomially bounded operator is similar to a contraction was posed by Halmos $[\mathrm{H}]$ and was answered in the negative by Pisier [Pi]. In particular, the sequence of finite dimensional operators which is uniformly polynomially bounded, but is not uniformly completely polynomially bounded was constructed in [Pi] (see also [DP]; see [Pa], [Pi], or [DP] for the definition of completely polynomially boundedness).

In [G16, Theorem 6.3], a perturbation of the sequence described above was constructed. The obtained perturbed sequence was used to construct examples of cyclic polynomially bounded operators which are not similar to contractions ([G16, Theorem 7.1 and Sec. 2]). In this paper, a slight modification of [G16, Theorem 7.1] is given which allows to construct a cyclic absolutely continuous polynomially bounded operator $T_{0}$ such that $T_{0}$ is not

2010 Mathematics Subject Classification. Primary 47A60; Secondary 47A65, 47A16, $47 \mathrm{~A} 20$.

Key words and phrases. Polynomially bounded operator, similarity, contraction, unilateral shift, isometry, $C_{0}$-contraction, $C_{0}$-operator. 
similar to a contraction and $\omega_{a}\left(T_{0}\right)=\mathbb{O}$, where $a>0$ and $\omega_{a}(z)=\exp \left(a \frac{z+1}{z-1}\right)$ $(z \in \mathbb{D})$ (Theorem 3.7).

We need the following notations and definitions.

Let $\mathcal{H}$ be a (complex, separable) Hilbert space, and let $\mathcal{M}$ be its (linear, closed) subspace. By $I_{\mathcal{H}}$ and $P_{\mathcal{M}}$ the identical operator on $\mathcal{H}$ and the orthogonal projection from $\mathcal{H}$ onto $\mathcal{M}$ are denoted, respectively.

For an operator $T: \mathcal{H} \rightarrow \mathcal{H}$, a subspace $\mathcal{M}$ of $\mathcal{H}$ is called invariant subspace of $T$, if $T \mathcal{M} \subset \mathcal{M}$. The complete lattice of all invariant subspaces of $T$ is denoted by Lat $T$.

The multiplicity $\mu_{T}$ of an operator $T: \mathcal{H} \rightarrow \mathcal{H}$ is the minimum dimension of its reproducing subspaces:

$$
\mu_{T}=\min \left\{\operatorname{dim} E: E \subset \mathcal{H}, \bigvee_{n=0}^{\infty} T^{n} E=\mathcal{H}\right\} .
$$

An operator $T$ is called cyclic, if $\mu_{T}=1$.

It is well known and easy to see that if $\mathcal{M} \in \operatorname{Lat} T$, then

$$
\mu_{P_{\left.\mathcal{M}^{\perp} T\right|_{\mathcal{M}} \perp}} \leq \mu_{T} \leq \mu_{\left.T\right|_{\mathcal{M}}}+\mu_{\left.P_{\mathcal{M}^{\perp}} T\right|_{\mathcal{M}^{\perp}}}
$$

(see, for example, [N, II.D.2.3.1]).

Let $T$ and $R$ be operators on Hilbert spaces $\mathcal{H}$ and $\mathcal{K}$, respectively, and let $X: \mathcal{H} \rightarrow \mathcal{K}$ be a (linear, bounded) operator such that $X$ intertwines $T$ and $R$, that is, $X T=R X$. If $X$ is unitary, then $T$ and $R$ are called unitarily equivalent, in notation: $T \cong R$. If $X$ is invertible, that is, $X$ has the bounded inverse $X^{-1}$, then $T$ and $R$ are called similar, in notation: $T \approx R$. If $X$ is a quasiaffinity, that is, $\operatorname{ker} X=\{0\}$ and $\operatorname{clos} X \mathcal{H}=\mathcal{K}$, then $T$ is called a quasiaffine transform of $R$, in notation: $T \prec R$. If $T \prec R$ and $R \prec T$, then $T$ and $R$ are called quasisimilar, in notation: $T \sim R$. If $\operatorname{clos} X \mathcal{H}=\mathcal{K}$, we write $T \stackrel{d}{\prec} R$. It follows immediately from the definition of the relation $\stackrel{d}{\prec}$ that if $T \stackrel{d}{\prec} R$, then $\mu_{R} \leq \mu_{T}$.

If $T, R$, and $X$ are operators such that $X T=R X$, and $\mathcal{M} \in \operatorname{Lat} T$, then $\operatorname{clos} X \mathcal{M} \in$ Lat $R$. The mapping

$$
\mathcal{J}_{X}: \text { Lat } T \rightarrow \text { Lat } R, \quad \mathcal{J}_{X} \mathcal{M}=\operatorname{clos} X \mathcal{M}, \quad \mathcal{M} \in \operatorname{Lat} T,
$$

is a lattice isomorphism if and only if it is a bijection, see [B, VII.1.19].

Symbols $\mathbb{D}$ and $\mathbb{T}$ denote the open unit disc and the unit circle, respectively. The normalized Lebesgue measure on $\mathbb{T}$ is denoted by $m$. The symbol $H^{\infty}$ denotes the Banach algebra of all bounded analytic functions in $\mathbb{D}$. The uniform norm on $\mathbb{D}$ is denoted by the symbol $\|\cdot\|_{\infty}$. The symbol $H^{2}$ denotes the Hardy space on $\mathbb{D}, L^{2}=L^{2}(\mathbb{T}, m), H_{-}^{2}=L^{2} \ominus H^{2}$. The symbol $P_{-}$denotes the orthogonal projection from $L^{2}$ onto $H_{-}^{2}$. The simple unilateral and bilateral shifts $S$ and $U_{\mathbb{T}}$ are the operators of multiplication by the independent variable on $H^{2}$ and on $L^{2}$, respectively. It is well known that $\mu_{S}=1$ and $\mu_{U_{\mathbb{T}}}=1$. For an inner function $\theta \in H^{\infty}$ the subspace $\theta H^{2}$ is invariant for $S$, put

$$
\mathcal{K}_{\theta}=H^{2} \ominus \theta H^{2} \quad \text { and } \quad T_{\theta}=\left.P_{\mathcal{K}_{\theta}} S\right|_{\mathcal{K}_{\theta}} .
$$

By $(1.1), \mu_{T_{\theta}}=1$. 
If $T$ is a polynomially bounded operator, then $T=T_{a} \dot{+} T_{s}$, where $T_{a}$ is an absolutely continuous (a.c.) polynomially bounded operator, that is, $H^{\infty}$-functional calculus is well defined for $T_{a}$, and $T_{s}$ is similar to a singular unitary operator, see [M] or [K16]. (Although many results on polynomially bounded operators that will be used in the present paper were originally proved by Mlak, we will refer to [K16] for the convenience of references.) In the paper, absolutely continuous polynomially bounded operators are considered. Clearly, $S$ and $U_{\mathbb{T}}$ are contraction, and, consequently, polynomially bounded. It is well known that $S$ and $U_{\mathbb{T}}$ are a.c.. An a.c. polynomially bounded operator $T$ is called a $C_{0}$-operator, if there exists $\varphi \in H^{\infty}$ such that $\varphi(T)=\mathbb{O}$, see $[\mathrm{BP}]$; if a $C_{0}$-operator is a contraction, it is called a $C_{0^{-}}$contraction, see $[\mathrm{SFBK}]$ and $[\mathrm{B}]$. For an inner function $\theta, T_{\theta}$ is a $C_{0^{-}}$ contraction, because $\theta\left(T_{\theta}\right)=\mathbb{O}$.

The paper is organized as follows. In Sec. 2 polynomially bounded operators that are quasiaffine transforms of the unilateral shift $S$ are considered. In many results is assumed that the operator $T$ under consideration has an invariant subspace $\mathcal{M}$ such that $\left.T\right|_{\mathcal{M}} \approx S$. If $T \prec S$ and $T$ is a contraction, then $T$ always has such invariant subspace (a particular case of [K07], see also [SFBK, Theorem IX.3.5]). The author does not know if this result can be generalized on polynomially bounded operators. In Sec. 3 Theorem 2.4 is applied to construct the polynomially bounded operator $T_{0}$ such that $T_{0}$ is not similar to a contraction and $\omega_{a}\left(T_{0}\right)=\mathbb{O}$, where $a>0$ and $\omega_{a}(z)=\exp \left(a \frac{z+1}{z-1}\right)(z \in \mathbb{D})$. (All subsequent results from Sec. 2 are not used in Sec. 3.)

\section{On Operators that aRe QUASIAFFine transforms of A UNILATERAL SHIFT}

An operator $T$ is called power bounded, if $\sup _{n \geq 0}\left\|T^{n}\right\|<\infty$. For a power bounded operator $T$ the isometric asymptote $(Y, V)$ is defined in [K89]. Here $V$ is an isometry, and $Y$ is an intertwining mapping: $Y T=V Y$. When it will be convenient, we will called by the isometric asymptote of $T$ the isometry $V$ itself, while $Y$ will be called the canonical intertwining mapping (sf. [K89] and $[\mathrm{K} 16])$.

Let $\mathcal{M} \in \operatorname{Lat} T$. It follows immediately from the construction of the isometric asymptote and [K89, Theorem 1] that $\left.T\right|_{\mathcal{M}}$ is similar to an isometry if and only if $\left.Y\right|_{\mathcal{M}}$ is left invertible, i.e., there exists $c>0$ such that $\|Y x\| \geq c\|x\|$ for every $x \in \mathcal{M}$. The unitary asymptote for a power bounded operator $T$ is the minimal unitary extension of its isometric asymptote, see [K89].

Clearly, a polynomially bounded operator is power bounded. The unitary asymptotes for polynomially bounded operators are considered in [K16].

In the following proposition, the idea from $[\mathrm{F}]$ is used. Since this proposition can be easily checked directly, its proof is omitted.

Proposition 2.1. Suppose that $T: \mathcal{H} \rightarrow \mathcal{H}, V: \mathcal{K} \rightarrow \mathcal{K}$, and $Y: \mathcal{H} \rightarrow \mathcal{K}$ are operators, and $Y T=V Y$. Furthermore, suppose that $\mathcal{M} \in \operatorname{Lat} T$ is such that $\left.Y\right|_{\mathcal{M}}$ is left invertible. Put $\mathcal{H}_{0}=\mathcal{H} \ominus \mathcal{M}, T_{0}=\left.P_{\mathcal{H}_{0}} T\right|_{\mathcal{H}_{0}}, \mathcal{K}_{1}=Y \mathcal{M}$, $\mathcal{K}_{0}=\mathcal{K} \ominus \mathcal{K}_{1}, Y_{2}=\left.P_{\mathcal{K}_{1}} Y\right|_{\mathcal{H}_{0}}, Y_{0}=\left.P_{\mathcal{K}_{0}} Y\right|_{\mathcal{H}_{0}}, V_{1}=\left.V\right|_{\mathcal{K}_{1}}, V_{2}=\left.P_{\mathcal{K}_{1}} V\right|_{\mathcal{K}_{0}}$, 

$V_{0}=\left.P_{\mathcal{K}_{0}} V\right|_{\mathcal{K}_{0}}$

$$
Z: \mathcal{M} \oplus \mathcal{H}_{0} \rightarrow \mathcal{K}_{1} \oplus \mathcal{H}_{0}, \quad Z=\left(\begin{array}{cc}
\left.Y\right|_{\mathcal{M}} & Y_{2} \\
\mathbb{O} & I_{\mathcal{H}_{0}}
\end{array}\right)
$$

and

$$
R: \mathcal{K}_{1} \oplus \mathcal{H}_{0} \rightarrow \mathcal{K}_{1} \oplus \mathcal{H}_{0}, \quad R=\left(\begin{array}{cc}
V_{1} & V_{2} Y_{0} \\
\mathbb{O} & T_{0}
\end{array}\right)
$$

Then $Y_{0} T_{0}=V_{0} Y_{0}, Z$ is invertible, $R=Z T Z^{-1}$, and

$$
\left(I_{\mathcal{K}_{1}} \oplus Y_{0}\right) R=V\left(I_{\mathcal{K}_{1}} \oplus Y_{0}\right) .
$$

Moreover, if $\operatorname{clos} Y \mathcal{H}=\mathcal{K}$, then $\operatorname{clos} Y_{0} \mathcal{H}_{0}=\mathcal{K}_{0}$, and if $\operatorname{ker} Y=\{0\}$, then $\operatorname{ker} Y_{0}=\{0\}$.

Lemma 2.2. Suppose that $C>0, g, \theta, \omega \in H^{\infty}, \theta$ is inner, and

$$
\left\|P_{-} \bar{\theta} \omega u\right\| \leq C\|g u\| \quad \text { for every } u \in H^{2} .
$$

Furthermore, suppose that $\mathcal{H}_{0}$ is a Hilbert space, and $X_{0}: \mathcal{K}_{\theta} \rightarrow \mathcal{H}_{0}$ is an operator. Put

$$
X: H^{2} \rightarrow \theta H^{2} \oplus \mathcal{H}_{0}, \quad X=\left(\begin{array}{cc}
\left.g(S)\right|_{\theta H^{2}} & \left.P_{\theta H^{2}} g(S)\right|_{\mathcal{K}_{\theta}} \\
\mathbb{O} & X_{0}
\end{array}\right) .
$$

Then there exists $C_{1}>0$ (which depends on $C,\|g\|_{\infty},\|\omega\|_{\infty}$, and $\left\|X_{0}\right\|$ ) such that

$$
\|X \omega u\| \leq C_{1}\|g u\| \quad \text { for every } u \in H^{2} .
$$

Proof. Let $u \in H^{2}$. We have

$$
X \omega u=\left(g P_{\theta H^{2}} \omega u+P_{\theta H^{2}} g P_{\mathcal{K}_{\theta}} \omega u\right) \oplus X_{0} P_{\mathcal{K}_{\theta}} \omega u .
$$

Therefore,

$$
\|X \omega u\|^{2}=\left\|g P_{\theta H^{2}} \omega u+P_{\theta H^{2}} g P_{\mathcal{K}_{\theta}} \omega u\right\|^{2}+\left\|X_{0} P_{\mathcal{K}_{\theta}} \omega u\right\|^{2} .
$$

We have $g P_{\theta H^{2}} \omega u+P_{\theta H^{2}} g P_{\mathcal{K}_{\theta}} \omega u=g \omega u-P_{\mathcal{K}_{\theta}} g P_{\mathcal{K}_{\theta}} \omega u$, $\left\|P_{\mathcal{K}_{\theta}} g P_{\mathcal{K}_{\theta}} \omega u\right\| \leq\|g\|_{\infty}\left\|P_{\mathcal{K}_{\theta}} \omega u\right\|, \quad$ and $\quad\left\|P_{\mathcal{K}_{\theta}} \omega u\right\|=\left\|\theta P_{-} \bar{\theta} \omega u\right\|=\left\|P_{-} \bar{\theta} \omega u\right\|$.

Therefore,

$$
\begin{aligned}
\| g P_{\theta H^{2}} \omega u & +P_{\theta H^{2}} g P_{\mathcal{K}_{\theta}} \omega u \|^{2} \leq\left(\|g \omega u\|+\left\|P_{\mathcal{K}_{\theta}} g P_{\mathcal{K}_{\theta}} \omega u\right\|\right)^{2} \\
& \leq 2\left(\|g \omega u\|^{2}+\left\|P_{\mathcal{K}_{\theta}} g P_{\mathcal{K}_{\theta}} \omega u\right\|^{2}\right) \leq 2\left(\|\omega\|_{\infty}^{2}\|g u\|^{2}+\|g\|_{\infty}^{2}\left\|P_{-} \bar{\theta} \omega u\right\|^{2}\right) .
\end{aligned}
$$

Thus,

$$
\begin{aligned}
\|X \omega u\|^{2} & \leq 2\left(\|\omega\|_{\infty}^{2}\|g u\|^{2}+\|g\|_{\infty}^{2}\left\|P_{-} \bar{\theta} \omega u\right\|^{2}\right)+\left\|X_{0}\right\|^{2}\left\|P_{-} \bar{\theta} \omega u\right\|^{2} \\
& =2\|\omega\|_{\infty}^{2}\|g u\|^{2}+\left(2\|g\|_{\infty}^{2}+\left\|X_{0}\right\|^{2}\right)\left\|P_{-} \bar{\theta} \omega u\right\|^{2} .
\end{aligned}
$$

It remains to apply $(2.1)$.

In the following lemma, only part (i) is used in the sequel.

Lemma 2.3. Suppose that $g, \theta, \omega \in H^{\infty}$, and $\theta$ is inner.

(i) If there exist $\eta, h \in H^{\infty}$ such that

$$
\omega=g \eta+\theta h,
$$

then there exists $C>0$ such that (2.1) is fulfilled. 
(ii) If $g$ is outer and (2.1) is fulfilled with some $C>0$, then there exist $\eta$, $h \in H^{\infty}$ such that (2.3) is fulfilled.

Proof. (i) Let $u \in H^{2}$. We have $P_{-} \bar{\theta} \omega u=P_{-} \bar{\theta}(g \eta+\theta h) u=P_{-} \bar{\theta} g \eta u$. Therefore, $\left\|P_{-} \bar{\theta} \omega u\right\| \leq\|g \eta u\| \leq\|\eta\|_{\infty}\|g u\|$.

(ii) For $\delta>0$ define an outer function $\psi_{\delta}$ by the formula

$$
\left|\psi_{\delta}(\zeta)\right|= \begin{cases}1 /|g(\zeta)|, & \text { if }|g(\zeta)| \geq \delta \\ 1, & \text { if }|g(\zeta)|<\delta\end{cases}
$$

Since $g$ is outer,

$$
\left(g \psi_{\delta}\right)(z) \rightarrow 1 \quad \text { for every } z \in \mathbb{D}, \quad \text { when } \delta \rightarrow 0 .
$$

Clearly, $\left\|g \psi_{\delta}\right\|_{\infty} \leq \max \left(\|g\|_{\infty}, 1\right)$. Set $C_{1}=\max \left(\|g\|_{\infty}, 1\right)$. Applying (2.1) with $\psi_{\delta} u$ instead of $u$, we obtain that

$$
\sup _{u \in H^{2},\|u\| \leq 1}\left\|P_{-} \bar{\theta} \omega \psi_{\delta} u\right\| \leq C C_{1} \quad \text { for every } \delta>0 .
$$

By the Nehari theorem (see, for example, [N, Theorem I.B.1.3.2]), there exist $\varphi_{\delta} \in H^{\infty}$ such that

$$
\left\|\bar{\theta} \omega \psi_{\delta}-\varphi_{\delta}\right\|_{\infty} \leq C C_{1} \quad \text { for every } \delta>0 .
$$

We have

$$
\begin{gathered}
\left\|g \varphi_{\delta}\right\|_{\infty}=\left\|\theta g \varphi_{\delta}\right\|_{\infty} \leq\left\|\theta g \varphi_{\delta}-\omega g \psi_{\delta}\right\|_{\infty}+\left\|\omega g \psi_{\delta}\right\|_{\infty} \leq\|g\|_{\infty} C C_{1}+\|\omega\|_{\infty} C_{1} \\
\text { for every } \delta>0 .
\end{gathered}
$$

By the Vitali-Montel theorem, there exist a sequence $\delta_{j} \rightarrow_{j} 0$ and $h, \eta \in H^{\infty}$ such that

$$
\left(g \varphi_{\delta_{j}}\right)(z) \rightarrow_{j} h(z) \text { and }\left(\omega \psi_{\delta_{j}}-\theta \varphi_{\delta_{j}}\right)(z) \rightarrow_{j} \eta(z) \text { for every } z \in \mathbb{D} .
$$

It follows from (2.4) and (2.5) that $\omega-\theta h=g \eta$.

Theorem 2.4. Suppose that $g, \eta, h, \theta, \omega \in H^{\infty}, \theta$ and $\omega$ are inner, and $\omega=g \eta+\theta h$. Furthermore, suppose that $\mathcal{H}_{0}$ is a Hilbert space, $T_{0}: \mathcal{H}_{0} \rightarrow \mathcal{H}_{0}$, $X_{0}: \mathcal{K}_{\theta} \rightarrow \mathcal{H}_{0}, Y_{0}: \mathcal{H}_{0} \rightarrow \mathcal{K}_{\theta}$ are operators, $Y_{0} X_{0}=g\left(T_{\theta}\right), X_{0} T_{\theta}=T_{0} X_{0}$, $Y_{0} T_{0}=T_{\theta} Y_{0}$. Define $X$ by $(2.2)$, put $Y=I_{\theta H^{2}} \oplus Y_{0}$,

$$
T: \theta H^{2} \oplus \mathcal{H}_{0} \rightarrow \theta H^{2} \oplus \mathcal{H}_{0}, \quad T=\left(\begin{array}{cc}
\left.S\right|_{\theta H^{2}} & \left.P_{\theta H^{2}} S\right|_{\mathcal{K}_{\theta}} Y_{0} \\
\mathbb{O} & T_{0}
\end{array}\right),
$$

and $\mathcal{M}=\operatorname{clos} X \omega H^{2}$. Then $\mathcal{M} \in \operatorname{Lat} T,\left.T\right|_{\mathcal{M}} \approx S$, and $Y \mathcal{M}=\vartheta \omega H^{2}$, where $\vartheta$ is the inner factor of $g$. Moreover,

$$
X S=T X, \quad Y T=S Y, \quad \text { and } \quad Y X=g(S) .
$$

Proof. The equalities (2.7) follow immediately from the definitions of $T, X$, and $Y$. The inclusion $\mathcal{M} \in$ Lat $T$ follows from the first equality in (2.7). By Lemma 2.3, (2.1) is fulfilled. We will show that there exists $c>0$ such that

$$
\|Y y\| \geq c\|y\| \quad \text { for every } y \in \mathcal{M} \text {. }
$$

It is sufficient to prove (2.8) for $y=X \omega u$, where $u \in H^{2}$, due to the definition of $\mathcal{M}$ and the continuity of $Y$. For such $y$, taking into account 
the third equality in (2.7) and the assumption that $\omega$ is inner, the condition (2.8) can be rewritten as follows:

$$
\|g u\| \geq c\|X \omega u\| \quad \text { for every } u \in H^{2} .
$$

This estimate is fulfilled by Lemma 2.2. Thus, (2.8) is proved.

By (2.8), the operator $\left.Y\right|_{\mathcal{M}}: \mathcal{M} \rightarrow Y \mathcal{M}$ is invertible. By the second equality in (2.7), $\left.Y\right|_{\mathcal{M}}$ realizes the relation $\left.\left.T\right|_{\mathcal{M}} \approx S\right|_{Y \mathcal{M}}$. We have

$$
Y \mathcal{M}=Y \operatorname{clos} X \omega H^{2}=\operatorname{clos} Y X \omega H^{2}=\operatorname{clos} g(S) \omega H^{2}=\vartheta \omega H^{2},
$$

where $\vartheta$ is the inner factor of $g$. Clearly, $\left.S\right|_{\vartheta \omega H^{2}} \cong S$.

The following simple lemma can be considered as a partial inverse to Theorem 2.4.

Lemma 2.5. Suppose $g, \eta, h, \omega \in H^{\infty}, \omega$ is inner, and $1=g \eta+\omega h$. Furthermore, suppose $T: \mathcal{H} \rightarrow \mathcal{H}$ is a polynomially bounded operator, $Y: \mathcal{H} \rightarrow$ $H^{2}$ is a quasiaffinity, $(Y, S)$ is the isometric asymptote of $T, X: H^{2} \rightarrow \mathcal{H}$ is an operator such that $Y X=g(S)$. Set $\mathcal{M}=\operatorname{clos} \omega(T) \mathcal{H}$. If $\left.T\right|_{\mathcal{M}} \approx S$, then $T \approx S$.

Proof. By assumption, $Y$ realizes the relation $T \prec S$. By [M] or [K16, Proposition 16], $T$ is a.c.. Thus, the operator $\omega(T)$ is well defined. Since $Y \omega(T)=\omega(S) Y$ and $\operatorname{clos} Y \mathcal{H}=H^{2}$, we conclude that $\operatorname{clos} Y \mathcal{M}=\omega H^{2}$. Suppose that $\left.T\right|_{\mathcal{M}} \approx S$. By [K89, Theorem 1], $\left.Y\right|_{\mathcal{M}}$ is left invertible, in particular, $Y \mathcal{M}=\operatorname{clos} Y \mathcal{M}=\omega H^{2}$. Let $u \in H^{2}$. There exists $x \in \mathcal{M}$ such that $\omega u=Y x$. We have

$$
\begin{aligned}
u & =(g \eta+\omega h) u=g(S)(\eta u)+h(S)(\omega u)=(Y X)(\eta u)+h(S) Y x \\
& =Y(X(\eta u)+h(T) x) .
\end{aligned}
$$

We conclude that $Y \mathcal{H}=H^{2}$. Since $\operatorname{ker} Y=\{0\}, Y$ is invertible by the Closed Graph Theorem.

The following lemma is Nevanlinna's theorem, see [N, Corollary I.B.3.3.2].

Lemma 2.6 (Nevanlinna's theorem). Suppose that $\theta, g, \eta \in H^{\infty},\|g \eta\|_{\infty}<$ 1 , and $\theta$ is an inner function. Then there exists an inner function $\omega \in H^{\infty}$ such that $\omega-g \eta \in \theta H^{\infty}$.

The following lemma is well known, see, for example, [S, Lemma 2] or [SFBK, Proposition X.1.1].

Lemma 2.7. Suppose that $\gamma, \theta, f \in H^{\infty}, \gamma$ and $\theta$ are inner, $\theta$ and the inner factor of $f$ are relatively prime. Then there exists $t \in \mathbb{C}$ such that $\gamma$ and the inner factor of $f+t \theta$ are relatively prime.

Lemma 2.8. Suppose that $\gamma, \theta \in H^{\infty}$ are inner functions, $T_{0}$ is a polynomially bounded operator, $Y_{0}$ is a quasiaffinity, and $Y_{0} T_{0}=T_{\theta} Y_{0}$. Then there exist a quasiaffinity $X_{0}$ and $g \in H^{\infty}$ such that $X_{0} T_{\theta}=T_{0} X_{0}, Y_{0} X_{0}=g\left(T_{\theta}\right)$, and $\gamma$ and the inner factor of $g$ are relatively prime.

Proof. By [BP], there exists a contraction $R_{0}$ such that $R_{0} \prec T_{0}$. From the relations $R_{0} \prec T_{0} \prec T_{\theta}$ and the fact that $T_{\theta}$ is a $C_{0^{-}}$-contraction we conclude that $T_{\theta} \prec R_{0}$ by [SFBK, Theorem X.5.7] or [B, Proposition III.5.32]. We obtain that $T_{0} \sim T_{\theta}$. Denote by $X_{0}$ the quasiaffinity which realizes the 
relation $T_{\theta} \prec T_{0}$. We have $Y_{0} X_{0} \in\left\{T_{\theta}\right\}^{\prime}$. By [SFBK, Theorem X.2.10] or [B, Proposition III.1.21], there exists $f \in H^{\infty}$ such that $Y_{0} X_{0}=f\left(T_{\theta}\right)$. Since $f\left(T_{\theta}\right)$ is a quasiaffinity, $\theta$ and the inner factor of $f$ are relatively prime. By Lemma 2.7, there exists $t \in \mathbb{C}$ such that $\gamma$ and the inner factor of $f+t \theta$ are relatively prime. Put $g=f+t \theta$. Then $Y_{0} X_{0}=g\left(T_{\theta}\right)$.

Lemma 2.9. Suppose that $T$ is a polynomially bounded operator and $T \prec S$. Then $S$ is the isometric asymptote of $T$.

Proof. Since $T$ is polynomially bounded, by [BP], there exists a contraction $R$ such that $R \prec T$. Clearly, $R \prec S$. By [KS, Proposition 9] or [G12, Lemma 2.1], the isometric asymptote of $R$ is $S$. Denote by $Y_{R}$ and $Y_{T}$ the canonical intertwining mappings for $R$ and $T$, and by $X$ and $Y$ the quasiaffinities which realize the relations $R \prec T$ and $T \prec S$, respectively. Let $V_{T}$ be the isometric asymptote of $T$. By [K89, Theorem 1(a)], there exist operators $Z_{1}$ and $Z_{2}$ such that $Z_{1} S=V_{T} Z_{1}, Y_{T} X=Z_{1} Y_{R}, Z_{2} V_{T}=S Z_{2}$, and $Y=Z_{2} Y_{T}$. Since the ranges of $Y_{T}$ and $Y$ are dense, the ranges of $Z_{1}$ and $Z_{2}$ are also dense. We obtain $S \stackrel{d}{\prec} V_{T}$ and $V_{T} \stackrel{d}{\prec} S$. By [K16, Proposition 16], $T$ is a.c.. Therefore, $V_{T}$ is a.c.. Thus, $V_{T} \cong S$.

Theorem 2.10. Suppose that $T: \mathcal{H} \rightarrow \mathcal{H}$ is a polynomially bounded operator, $T \prec S, \mathcal{M} \in \operatorname{Lat} T$, and $\left.T\right|_{\mathcal{M}} \approx S$. Then there exist the operators $X_{1}, X_{2}: H^{2} \rightarrow \mathcal{H}, Y: \mathcal{H} \rightarrow H^{2}$, the functions $g_{1}, g_{2} \in H^{\infty}$ and $\mathcal{N} \in \operatorname{Lat} T$ such that

$$
Y T=S Y, \quad X_{k} S=T X_{k}, \quad Y X_{k}=g_{k}(S) \quad \text { and } \quad X_{k} Y=g_{k}(T), \quad k=1,2,
$$

the inner factors of $g_{1}$ and $g_{2}$ are relatively prime, $\left.T\right|_{\mathcal{N}} \approx S$, and $\mathcal{M} \vee \mathcal{N}=$ $\mathcal{H}$.

Proof. By Lemma 2.9, the isometric asymptote of $T$ is $S$. Denote by $Y$ the canonical intertwining mapping. Then $\left.Y\right|_{\mathcal{M}}$ is left invertible. Let $\theta \in H^{\infty}$ be an inner function such that $Y \mathcal{M}=\theta H^{2}$. Let $T_{0}$ and $Y_{0}$ be defined as in Proposition 2.1. Without loss of generality, we can suppose that $T$ has the form (2.6) and $Y=I_{\theta H^{2}} \oplus Y_{0}$. Then $Y_{0}$ is a quasiaffinity which realizes the relation $T_{0} \prec T_{\theta}$.

Denote by $X_{10}$ and $g_{1}$ the quasiaffinity and the function from Lemma 2.8 applied to $\gamma=\theta, \theta, T_{0}$, and $Y_{0}$. Denote by $\vartheta_{1}$ the inner factor of $g_{1}$. By Lemma 2.8, $\theta$ and $\vartheta_{1}$ are relatively prime. Denote by $X_{1}$ the operator defined by (2.2) applied to $g_{1}$ and $X_{10}$.

Take $\eta \in H^{\infty}$ such that $\left\|g_{1} \eta\right\|_{\infty}<1$, and $\theta$ and the inner factor of $\eta$ are relatively prime. By Lemma 2.6, there exist $\omega, h \in H^{\infty}$ such that $\omega$ is inner and $\omega=g_{1} \eta+\theta h$. Put $\mathcal{N}=\operatorname{clos} X_{1} \omega H^{2}$. By Theorem 2.4, $\mathcal{N} \in \operatorname{Lat} T$, $\left.T\right|_{\mathcal{N}} \approx S$, and $Y \mathcal{N}=\vartheta_{1} \omega H^{2}$. Furthermore, (2.7) is fulfilled for $T, X_{1}, Y$, and $g_{1}$. Note that $\theta$ and $\vartheta_{1} \omega$ are relatively prime.

Denote by $X_{20}$ and $g_{2}$ the quasiaffinity and the function from Lemma 2.8 applied to $\gamma=\vartheta_{1}, \theta, T_{0}$, and $Y_{0}$. Denote by $\vartheta_{2}$ the inner factor of $g_{2}$. By Lemma 2.8, $\vartheta_{1}$ and $\vartheta_{2}$ are relatively prime. Denote by $X_{2}$ the operator defined by (2.2) applied to $g_{2}$ and $X_{20}$. Then (2.7) is fulfilled for $T, X_{2}, Y$, and $g_{2}$. 
It is easy to see from (2.6) and (2.7) that

$$
g(T)=\left(\begin{array}{cc}
\left.g(S)\right|_{\theta H^{2}} & \left.P_{\theta H^{2}} g(S)\right|_{\mathcal{K}_{\theta}} Y_{0} \\
\mathbb{O} & g\left(T_{0}\right)
\end{array}\right)
$$

for every $g \in H^{\infty}$. The equations (2.9) follow from this equation and (2.7).

It is well known that if (2.9) are fulfilled, and the inner factors of $g_{1}$ and $g_{2}$ are relatively prime, then the mapping $\mathcal{J}_{Y}$ defined by (1.2) is a lattice isomorphism. We have

$$
\mathcal{J}_{Y}(\mathcal{M} \vee \mathcal{N})=\mathcal{J}_{Y} \mathcal{M} \vee \mathcal{J}_{Y} \mathcal{N}=\theta H^{2} \vee \vartheta_{1} \omega H^{2}=H^{2}
$$

(because $\theta$ and $\vartheta_{1} \omega$ are relatively prime). Therefore, $\mathcal{M} \vee \mathcal{N}=\mathcal{H}$.

Remark 2.11. If $T$ is a contraction such that $T \prec S$, then there exists $\mathcal{M} \in$ Lat $T$ such that $\left.T\right|_{\mathcal{M}} \approx S$ by [K07] or [SFBK, Theorem IX.3.5]. Thus, $T$ satisfies to the conditions of Theorem 2.10.

Lemma 2.12. Suppose that $T$ is a polynomially bounded operator, $Y$ is a quasiaffinity, and $Y T=S Y$. Then the mapping $\mathcal{J}_{Y}$ defined by (1.2) is a lattice isomorphism.

Proof. By [BP], there exists a contraction $R$ such that $R \prec T$. Clearly, $R \prec S$. By Remark 2.11, the conclusion of Theorem 2.10 is fulfilled for $R$. Using the relation $R \prec T$, it is easy to see that there exist operators $X_{1}, X_{2}: H^{2} \rightarrow \mathcal{H}$ and the functions $g_{1}, g_{2} \in H^{\infty}$ such that the inner factors of $g_{1}$ and $g_{2}$ are relatively prime and (2.9) is fulfilled for $T$ and $Y$. Since the inner factors of functions $g_{1}$ and $g_{2}$ are relatively prime, $\mathcal{J}_{Y}$ is a latticeisomorphism.

Remark 2.13. The relations (2.9) are proved for contractions $T$ such that $T \prec S$ in [G03, Corollary 2.5] in other way.

The following lemma follows from the definition of a.c. polynomially bounded operators (see [G17, Lemma 2.2] for the proof).

Lemma 2.14. Suppose that $T$ is a polynomially bounded operator, and $\mathcal{M} \in$ Lat $T$. Then $T$ is a.c. if and only if $\left.T\right|_{\mathcal{M}}$ and $\left.P_{\mathcal{M}^{\perp}} T\right|_{\mathcal{M}^{\perp}}$ are a.c..

The following lemma can be easily checked directly, therefore, its proof is omitted.

Lemma 2.15. Suppose that $T: \mathcal{H} \rightarrow \mathcal{H}$ is an operator, $\mathcal{M}, \mathcal{N} \in \operatorname{Lat} T$, and $\mathcal{H}=\mathcal{M} \vee \mathcal{N}$. Put $\mathcal{E}=\mathcal{M} \cap \mathcal{N}$. Then the quasiaffinity $\left.P_{\mathcal{M}^{\perp}}\right|_{\mathcal{N} \ominus \mathcal{E}}$ realizes the relation

$$
\left.\left.P_{\mathcal{N} \ominus \mathcal{E}} T\right|_{\mathcal{N} \ominus \mathcal{E}} \prec P_{\mathcal{M}^{\perp}} T\right|_{\mathcal{M}^{\perp}}
$$

The following proposition is closed to [T] and [KS, Proposition 20].

Proposition 2.16. Suppose that $T: \mathcal{H} \rightarrow \mathcal{H}$ is a polynomially bounded operator, $\mathcal{M}_{1}, \mathcal{M}_{2} \in \operatorname{Lat} T,\left.T\right|_{\mathcal{M}_{k}} \prec S, k=1,2, \mathcal{M}_{1} \vee \mathcal{M}_{2}=\mathcal{H}$ and $\mathcal{M}_{1} \cap \mathcal{M}_{2} \neq\{0\}$. Then $T$ is a.c., $T \stackrel{d}{\prec} S$, and $S$ is the isometric asymptote of $T$. Moreover, if $T \nprec S$, then $\left.T\right|_{\operatorname{ker} Y}$ is a $C_{0}$-operator, where $Y$ is a canonical intertwining mapping. 
Proof. Put $\mathcal{E}=\mathcal{M}_{1} \cap \mathcal{M}_{2}$. We can suppose that $\mathcal{E} \neq \mathcal{M}_{2}$. Denote by $X$ a quasiaffinity which realizes the relation $\left.T\right|_{\mathcal{M}_{2}} \prec S$. There exists an inner function $\theta \in H^{\infty}$ such that $\mathcal{J}_{X} \mathcal{E}=\theta H^{2}$, where $\mathcal{J}_{X}$ is defined by (1.2). Put

$$
T_{0}=\left.P_{\mathcal{M}_{2} \ominus \mathcal{E}} T\right|_{\mathcal{M}_{2} \ominus \mathcal{E}}, \quad T_{1}=\left.P_{\mathcal{M}_{1}^{\perp}} T\right|_{\mathcal{M}_{1}^{\perp}}, \quad \text { and } \quad X_{0}=\left.P_{\mathcal{K}_{\theta}} X_{0}\right|_{\mathcal{M}_{2} \ominus \mathcal{E}} .
$$

Clearly, $X_{0} T_{0}=T_{\theta} X_{0}$. Taking into account that $\mathcal{J}_{X}$ is a lattice isomorphism (by Lemma 2.12), it is easy to see that $X_{0}$ is a quasiaffinity. Therefore, $T_{0}$ is a.c. ([K16, Proposition 16]) and $\theta\left(T_{0}\right)=\mathbb{O}$.

By Lemma 2.15, $T_{0} \prec T_{1}$. By [M] or [K16, Proposition 16] and Lemma 2.14, $T$ is a.c.. Furthermore,

$$
\theta\left(T_{1}\right)=\mathbb{O} \text {. }
$$

Let $(Y, U)$ be the unitary asymptote of $T$. By [K89, Theorem 3] and Lemma $2.9, U \cong U_{\mathbb{T}}$, and $\left.Y\right|_{\mathcal{M}_{1}}$ realizes the relation $\left.\left.T\right|_{\mathcal{M}_{1}} \prec U_{\mathbb{T}}\right|_{\xi H^{2}}$ for some $\xi \in L^{\infty}$ such that $|\xi|=1$ a.e. with respect to $m$.

If clos $Y \mathcal{H}=L^{2}$, then $\left.P_{\xi H_{-}^{2}} Y\right|_{\mathcal{M}_{1}^{\perp}}$ realizes the relation $\left.T_{1} \stackrel{d}{\prec} P_{\xi H_{-}^{2}} U_{\mathbb{T}}\right|_{\xi H_{-}^{2}}$, which contradicts to (2.10). Thus, $\xi H^{2} \subset \operatorname{clos} Y \mathcal{H} \neq L^{2}$. Since $\operatorname{clos} Y \mathcal{H} \in$ Lat $U_{\mathbb{T}}$, we conclude that $\operatorname{clos} Y \mathcal{H}=\xi_{1} H^{2}$ for some $\xi_{1} \in L^{\infty}$ such that $\left|\xi_{1}\right|=1$ a.e. with respect to $m$. Since $\left.U_{\mathbb{T}}\right|_{\xi_{1} H^{2}} \cong S$, we conclude that $S$ is the isometric asymptote of $T$.

Put $\mathcal{E}_{0}=\operatorname{ker} Y$ and $\mathcal{E}_{1}=\operatorname{clos} P_{\mathcal{M}_{1}^{\perp}} \mathcal{E}_{0}$. Then $\mathcal{E}_{0} \in \operatorname{Lat} T$ and $\mathcal{E}_{0} \cap \mathcal{M}_{1}=$ $\{0\}$. Therefore, $\mathcal{E}_{1} \in \operatorname{Lat} T_{1}$ and $\left.P_{\mathcal{E}_{1}}\right|_{\mathcal{E}_{0}}$ realizes the relation $\left.\left.T\right|_{\mathcal{E}_{0}} \prec T_{1}\right|_{\mathcal{E}_{1}}$. By $(2.10), \theta\left(\left.T\right|_{\mathcal{E}_{0}}\right)=\mathbb{O}$. Thus, $\left.T\right|_{\mathcal{E}_{0}}$ is a $C_{0}$-operator.

\section{Existence of a polynomially bounded operator With the MINIMAL FUNCTION $\exp \left(a \frac{z+1}{z-1}\right)$ WHICH IS NOT SIMILAR TO A CONTRACTION}

For $\lambda \in \mathbb{D}$ denote by $b_{\lambda}$ a Blaschke factor: $b_{\lambda}(z)=\frac{|\lambda|}{\lambda} \frac{\lambda-z}{1-\bar{\lambda} z}, z \in \mathbb{D}$. For $w \in \mathbb{D}$ put

$$
\beta_{w}(z)=\frac{w-z}{1-\bar{w} z}, \quad z \in \mathbb{D} .
$$

Clearly, $\beta_{w} \circ \beta_{w}(z)=z$ for every $z \in \mathbb{D}$. For every $\varphi \in H^{\infty}$ we have $\varphi \circ \beta_{w} \in H^{\infty},\left\|\varphi \circ \beta_{w}\right\|_{\infty}=\|\varphi\|_{\infty}$, and

$$
b_{\lambda} \circ \beta_{w}=\zeta_{w, \lambda} b_{\beta_{w}(\lambda)}, \quad \text { where } \zeta_{w, \lambda} \in \mathbb{T} .
$$

For $a>0$ and $0<\alpha<1$ put

$$
\omega_{a}(z)=\exp \left(a \frac{z+1}{z-1}\right) \text { and } g_{\alpha}(z)=\exp \left(-\left(\frac{1+z}{1-z}\right)^{\alpha}\right), \quad z \in \mathbb{D} .
$$

We have $\omega_{a}, g_{\alpha} \in H^{\infty}, \omega_{a}$ is inner, and $g_{\alpha}$ is outer by [N, Example I.A.4.3.7, p. 71].

The following lemma can be easily proved by induction. Therefore, its proof is omitted.

Lemma 3.1. Let $a>0$, and let $0<\alpha<1$. Let $\omega_{a}$ and $g_{\alpha}$ be defined in (3.2). Let $n \in \mathbb{N}$. Then there exist functions $\psi_{\omega, n}, \psi_{g, n}$ analytic in $\mathbb{D}$ such that

$$
\omega_{a}^{(n)}=\omega_{a} \psi_{\omega, n} \quad \text { and } \quad g_{\alpha}^{(n)}=g_{\alpha} \psi_{g, n} .
$$


Furthermore,

$$
\psi_{\omega, n}(z)=\sum_{l=1}^{K_{\omega, n}} \frac{c_{\omega, n, l}}{(1-z)^{k_{\omega, n, l}}} \text { and } \psi_{g, n}(z)=\sum_{l=1}^{K_{g, n}} c_{g, n, l}\left(\frac{1+z}{1-z}\right)^{d_{1, l, n}}(1-z)^{d_{2, n, l}},
$$

where $K_{\omega, n}, K_{g, n}, k_{\omega, n, l} \in \mathbb{N}, c_{\omega, n, l}, c_{g, n, l}, d_{1, l, n}, d_{2, n, l} \in \mathbb{R}$.

Lemma 3.2. Let $a>0$, and let $0<\alpha<1$. Let $\omega_{a}$ and $g_{\alpha}$ be defined in (3.2). Put

$$
\begin{aligned}
& \psi_{g, n, k}=\frac{n !}{k !(n-k) !} \psi_{g, n-k}, \quad 1 \leq k \leq n, \\
& \psi_{g, k, k}=1, \quad \psi_{g, n, k}=0, \quad n \leq k-1, \quad \psi_{\omega, 0}=1 .
\end{aligned}
$$

For $M \in \mathbb{N}$ set $\Psi_{M}=\left[\psi_{g, n, k}\right]_{n, k=0}^{M-1}$ and define the functions $\kappa_{M, n}, 0 \leq n \leq$ $M-1$, by the formula

$$
\left[\kappa_{M, n}\right]_{n=0}^{M-1}=\frac{\omega_{a}}{g_{\alpha}} \cdot \Psi_{M}^{-1} \cdot\left[\psi_{\omega, n}\right]_{n=0}^{M-1} .
$$

Then $\kappa_{M, n}$ are functions analytic in $\mathbb{D}$, and $\kappa_{M, n}(z) \rightarrow 0$ when $z \in(0,1)$, $z \rightarrow 1$ for every $0 \leq n \leq M-1$.

Proof. We have $\Psi_{M}$ is a lower triangular matrix-function, and the elements of the main diagonal of $\Psi_{M}$ are equal to 1 . Therefore, $\Psi_{M}$ is invertible. The elements of the matrix-function $\Psi_{M}^{-1} \cdot\left[\psi_{\omega, n}\right]_{n=0}^{M-1}$ have the form

$$
\sum_{l=1}^{K} c_{l}\left(\frac{1+z}{1-z}\right)^{d_{1, l}}(1-z)^{d_{2, l}}
$$

for some $K \in \mathbb{N}$ and $c_{l}, d_{1, l}, d_{2, l} \in \mathbb{R}$ (depended on $\alpha, a, M$, and the indices of the considered element of the matrix).

Set $t=\frac{1+z}{1-z}$. Then $1-z=\frac{2}{1+t}$, and $\frac{\omega_{a}(z)}{g_{\alpha}(z)}=\exp \left(-a t+t^{\alpha}\right)$. Furthermore, $t \rightarrow+\infty$ when $z \in(0,1), z \rightarrow 1$. We have $t^{d_{1}}(1+t)^{d_{2}} \exp \left(-a t+t^{\alpha}\right) \rightarrow 0$ when $t \rightarrow+\infty$ for every $d_{1}, d_{2} \in \mathbb{R}$. The conclusion of the lemma follows from this relation.

The following lemma is an emphasizing of [G16, Lemma 3.5].

Lemma 3.3. Suppose that $\Lambda \subset \mathbb{D}$ is finite, $1 \leq k_{\lambda}<\infty$ for every $\lambda \in \Lambda$, and $B=\prod_{\lambda \in \Lambda} b_{\lambda}^{k_{\lambda}}$. Then there exists $C>0$ which depends on $B$ such that

$$
\operatorname{dist}\left(\varphi,\left(B \circ \beta_{w}\right) H^{\infty}\right) \leq C \max _{\lambda \in \Lambda, 0 \leq k \leq k_{\lambda}-1}\left|\varphi^{(k)}\left(\beta_{w}(\lambda)\right)\right|
$$

for every $\varphi \in H^{\infty}$ and every $w \in \mathbb{D}$.

Proof. We have $\operatorname{dist}\left(\varphi,\left(B \circ \beta_{w}\right) H^{\infty}\right)=\operatorname{dist}\left(\varphi \circ \beta_{w}, B H^{\infty}\right)$ for every $\varphi \in H^{\infty}$ and every $w \in \mathbb{D}$. By [G16, Lemma 3.5], there exists $C>0$ (which depends on $B$ ) such that

$$
\operatorname{dist}\left(\psi, B H^{\infty}\right) \leq C \max _{\lambda \in \Lambda, 0 \leq k \leq k_{\lambda}-1}\left|\psi^{(k)}(\lambda)\right| \text { for every } \psi \in H^{\infty} .
$$

We need to estimate $\left|\left(\varphi \circ \beta_{w}\right)^{(k)}(\lambda)\right|$ for $1 \leq k \leq k_{\lambda}-1$. By [G16, Lemma 3.3], there exist functions $c_{k l}: \mathbb{D} \rightarrow \mathbb{C}$ such that $\sup _{\mathbb{D}}\left|c_{k l}\right|<\infty$ for every 
$k \geq 1,0 \leq l \leq k-1$, and for every analytic function $\varphi: \mathbb{D} \rightarrow \mathbb{C}$ and every $w \in \mathbb{D}$

$$
\left(\varphi \circ \beta_{w}\right)^{(k)}(z)=\sum_{l=1}^{k} \varphi^{(l)}\left(\beta_{w}(z)\right) \frac{c_{k, k-l}(w)}{(1-\bar{w} z)^{k+l}} .
$$

Therefore,

$$
\begin{gathered}
\left|\left(\varphi \circ \beta_{w}\right)^{(k)}(\lambda)\right| \leq \sum_{l=1}^{k}\left|\varphi^{(l)}\left(\beta_{w}(\lambda)\right)\right| \frac{\left|c_{k, k-l}(w)\right|}{|1-\bar{w} \lambda|^{k+l}} \\
\leq \max _{\lambda \in \Lambda, 0 \leq k \leq k_{\lambda}-1}\left|\varphi^{(k)}\left(\beta_{w}(\lambda)\right)\right| \cdot \max _{\lambda \in \Lambda}\left(k_{\lambda}-1\right) \cdot \sup _{\substack{1 \leq l \leq k, 1 \leq k \leq k_{\lambda}-1 \\
w \in \mathbb{D}}} \frac{\left|c_{k, k-l}(w)\right|}{\left(1-\max _{\lambda \in \Lambda}|\lambda|\right)^{k+l}}
\end{gathered}
$$

for $1 \leq k \leq k_{\lambda}-1$.

Theorem 3.4. Suppose that $C>0, a>0,0<\alpha<1$, and $\left\{B_{N}\right\}_{N}$ is a sequence of finite Blaschke products with zeros from $(0,1)$. Then there exists a sequence $\left\{\rho_{N}\right\}_{N} \subset(0,1)$ such that for any $w \in\left[\rho_{N}, 1\right)$ there exists $\eta_{N, w} \in H^{\infty}$ such that

(3.3) $\operatorname{dist}\left(\eta_{N, w},\left(B_{N} \circ \beta_{w}\right) H^{\infty}\right) \leq C \quad$ and $\quad \omega_{a}-g_{\alpha} \eta_{N, w} \in\left(B_{N} \circ \beta_{w}\right) H^{\infty}$, where $\beta_{w}$ is defined in (3.1), and $\omega_{a}$ and $g_{\alpha}$ are defined in (3.2).

Proof. Let $N$ be fixed. Denote by $C_{N}$ a constant from Lemma 3.3 applied to $B_{N}=\prod_{\lambda \in \Lambda} b_{\lambda}^{k_{\lambda}}$. By Lemma 3.2, there exists $\rho_{1 N} \in(0,1)$ such that $\left|\kappa_{k_{\lambda}, k}(z)\right| \leq C / C_{N}$ for every $z \in\left[\rho_{1 N}, 1\right), 0 \leq k \leq k_{\lambda}-1, \lambda \in \Lambda$.

Since $\beta_{w}(\lambda) \rightarrow 1$ when $w \rightarrow 1$ for every $\lambda \in \mathbb{D}$, and $\beta_{w}(\lambda) \in(0,1)$ when $0<\lambda<w<1$, there exists $\rho_{N} \in(0,1)$ such that $\beta_{w}(\lambda) \in\left[\rho_{1 N}, 1\right)$ when $w \in\left[\rho_{N}, 1\right)$ for every $\lambda \in \Lambda$.

Since $\Lambda$ is a finite set, and $k_{\lambda}<\infty$ for every $\lambda \in \Lambda$, we have that for every $w \in \mathbb{D}$ there exists $\eta_{N, w} \in H^{\infty}$ such that

$$
\eta_{N, w}^{(k)}\left(\beta_{w}(\lambda)\right)=\kappa_{k_{\lambda}, k}\left(\beta_{w}(\lambda)\right) \text { for every } \lambda \in \Lambda \text { and } 0 \leq k \leq k_{\lambda}-1 .
$$

By Lemma 3.3, dist $\left(\eta_{N, w},\left(B \circ \beta_{w}\right) H^{\infty}\right) \leq C$ for $w \in\left[\rho_{N}, 1\right)$.

The inclusion in (3.3) is fulfilled if and only if

(3.5) $\omega_{a}^{(k)}\left(\beta_{w}(\lambda)\right)-\left(g_{\alpha} \eta_{N, w}\right)^{(k)}\left(\beta_{w}(\lambda)\right)=0$ for all $\lambda \in \Lambda, 0 \leq k \leq k_{\lambda}-1$.

Clearly,

$$
\left(g_{\alpha} \eta_{N, w}\right)^{(n)}(z)=\sum_{k=0}^{n} \frac{n !}{k !(n-k) !} g_{\alpha}^{(n-k)}(z) \eta_{N, w}^{(k)}(z) \text { for } n \in \mathbb{N} \text { and } z \in \mathbb{D} \text {. }
$$

Therefore,

$$
\left[\left(g_{\alpha} \eta_{N, w}\right)^{(k)}(z)\right]_{k=0}^{k_{\lambda}-1}=g_{\alpha}(z) \cdot \Psi_{k_{\lambda}}(z) \cdot\left[\eta_{N, w}^{(k)}(z)\right]_{k=0}^{k_{\lambda}-1}
$$

for every $z \in \mathbb{D}$, where $\Psi_{k_{\lambda}}$ is defined in Lemma 3.2. By (3.4) and Lemmas 3.1 and 3.2 ,

$$
\begin{aligned}
{\left[\left(g_{\alpha} \eta_{N, w}\right)^{(k)}(z)\right]_{k=0}^{k_{\lambda}-1} } & =g_{\alpha}(z) \cdot \Psi_{k_{\lambda}}(z) \cdot\left[\kappa_{k_{\lambda}, k}(z)\right]_{k=0}^{k_{\lambda}-1}=\omega_{a}(z) \cdot\left[\psi_{\omega, k}(z)\right]_{k=0}^{k_{\lambda}-1} \\
& =\left[\omega_{a}^{(k)}(z)\right]_{k=0}^{k_{\lambda}-1} \text { when } z=\beta_{w}(\lambda), \lambda \in \Lambda .
\end{aligned}
$$

Thus, the equalities (3.5) are fulfilled, and the inclusion in (3.3) is proved. 
The following theorem is a version of [G16, Theorem 3.8]. The condition (3.6) from the theorem is the generalized Carleson condition, see [N, Theorem II.C.3.2.14, p. 164].

Theorem 3.5. Suppose that $C>0,\left\{\rho_{N}\right\}_{N} \subset(0,1), g \in H^{\infty}$, and $g^{(k)}(r) \rightarrow$ 0 when $r \in(0,1), r \rightarrow 1$, for every $k \geq 0$. Furthermore, suppose that $B_{N}$ are finite Blaschke products with zeros from $(0,1), \varphi_{N} \in H^{\infty}, \varphi_{N}(\lambda) \neq 0$ for every $\lambda \in \mathbb{D}$ such that $B_{N}(\lambda)=0$, for every index $N$. Then there exist $\delta>0$ and sequences of $w_{N} \in\left[\rho_{N}, 1\right)$, of $\zeta_{N} \in \mathbb{T}$, and of $\psi_{N} \in H^{\infty}$ such that the product $\prod_{N} \zeta_{N} B_{N} \circ \beta_{w_{N}}$ converges,

$$
\left|\prod_{N} \zeta_{N}\left(B_{N} \circ \beta_{w_{N}}\right)(z)\right| \geq \delta \inf _{N}\left|\left(B_{N} \circ \beta_{w_{N}}\right)(z)\right| \quad \text { for every } z \in \mathbb{D}
$$

$g-\psi_{N} \cdot \varphi_{N} \circ \beta_{w_{N}} \in\left(B_{N} \circ \beta_{w_{N}}\right) H^{\infty}$, and $\operatorname{dist}\left(\psi_{N},\left(B_{N} \circ \beta_{w_{N}}\right) H^{\infty}\right) \leq C$.

Proof. The proof of [G16, Theorem 3.8] is beginned from the definition of the sequence $\left\{r_{N}\right\}_{N} \subset(0,1)$. To prove the present theorem, it needs to replace $r_{N}$ by $\max \left(r_{N}, \rho_{N}\right)$ and to repeat the proof of [G16, Theorem 3.8].

The following theorem is a version of [G16, Theorem 7.1].

Theorem 3.6. Let $a>0$, and let $0<\alpha<1$. Let $\omega_{a}$ and $g_{\alpha}$ be defined in (3.2). Then there exist a function $\eta \in H^{\infty}$, an operator $T: \mathcal{H} \rightarrow \mathcal{H}$, a Blaschke product $B$ with zeros from $(0,1)$, and quasiaffinities $X: \mathcal{H} \rightarrow \mathcal{K}_{B}$, $Y: \mathcal{K}_{B} \rightarrow \mathcal{H}$ such that

$$
\omega_{a}-g_{\alpha} \eta \in B H^{\infty}
$$

$T$ is polynomially bounded, $T$ is not similar to a contraction,

$$
X T=T_{B} X, \quad Y T_{B}=T Y, \quad \text { and } \quad X Y=g_{\alpha}\left(T_{B}\right),
$$

where $T_{B}$ is defined in (1.3).

Proof. Let $\left\{T_{N}\right\}_{N}$ and $\left\{B_{N}\right\}_{N}$ be the sequences of operators and of finite Blaschke products with zeros from $(0,1)$ from [G16, Theorem 6.3], respectively. Let $C>0$ be fixed. Denote by $\mathcal{H}_{N}$ the finite dimensional spaces on which $T_{N}$ acts. There exist invertible operators $X_{N}: \mathcal{H}_{N} \rightarrow \mathcal{K}_{B_{N}}$, $Y_{N}: \mathcal{K}_{B_{N}} \rightarrow \mathcal{H}_{N}$ such that $X_{N} T_{N}=T_{B_{N}} X_{N}, Y_{N} T_{B_{N}}=T_{N} Y_{N},\left\|X_{N}\right\| \leq C$, $\left\|Y_{N}\right\| \leq C$. By [SFBK, Theorem X.2.10], there exist functions $\varphi_{N} \in H^{\infty}$ such that $X_{N} Y_{N}=\varphi_{N}\left(T_{B_{N}}\right)$. Note that $\varphi_{N}(\lambda) \neq 0$ for every $\lambda \in \mathbb{D}$ such that $B_{N}(\lambda)=0$, for every index $N$.

Let $\left\{\rho_{N}\right\}_{N}$ be the sequence from Theorem 3.4 applied to $C, a, \alpha$, and $\left\{B_{N}\right\}_{N}$. Applying Theorem 3.5 to $C,\left\{\rho_{N}\right\}_{N}, g_{\alpha}$, sequences of $B_{N}$ and of $\varphi_{N}$ we obtain $\delta>0$ and sequences of $w_{N} \in\left[\rho_{N}, 1\right)$, of $\zeta_{N} \in \mathbb{T}$ and of $\psi_{N} \in H^{\infty}$ which satisfy the conclusion of Theorem 3.5. Put

$$
B=\prod_{N} \zeta_{N} B_{N} \circ \beta_{w_{N}} \text { and } T=\bigoplus_{N} \beta_{w_{N}}\left(T_{N}\right) .
$$

By [G16, Theorem 6.3] and [G16, Corollary 1.2], $T$ is polynomially bounded, and $T$ is not similar to a contraction.

Let $\eta_{N, w_{N}}$ be from Theorem 3.4. Then (3.3) is fulfilled for every $N$ with $w=w_{N}$. By (3.6), (3.3), and [N, Theorem II.C.3.2.14, p. 164], there exists 
a function $\eta \in H^{\infty}$ such that $\eta-\eta_{N, w_{N}} \in\left(B_{N} \circ \beta_{w_{N}}\right) H^{\infty}$ for every $N$. We have

$$
\omega_{a}-g_{\alpha} \eta=\omega_{a}-g_{\alpha} \eta_{N, w_{N}}+g_{\alpha}\left(\eta_{N, w_{N}}-\eta\right) \in\left(B_{N} \circ \beta_{w_{N}}\right) H^{\infty}
$$

for every $N$ due to (3.3). Thus, (3.7) is proved.

Equalities (3.8) are proved exactly as in the proof of [G16, Theorem 7.1].

Theorem 3.7. Let $a>0$, and let $\omega_{a}$ be defined in (3.2). Then there exists a cyclic a.c. polynomially bounded operator $T_{0}$ such that $T_{0}$ is not similar to a contraction and $\omega_{a}\left(T_{0}\right)=\mathbb{O}$.

Proof. Take $0<\alpha<1$, and apply Theorem 3.6 to $a$ and $\alpha$. Denote the polynomially bounded operator and quasiaffinities constructed in Theorem 3.6 by $T_{10}, X_{10}$, and $Y_{10}$, respectively. Define $X$ and $T$ by (2.2) and (2.6), respectively, with $\theta=B$, where $B$ is the Blaschke product obtained in Theorem 3.6. By [G16, Corollary 2.3], $T$ is polynomially bounded, and $T$ is not similar to a contraction. Set $Y=I_{B H^{2}} \oplus Y_{10}$. Clearly, $Y$ is a quasiaffinity. Since $g_{\alpha}$ is outer, $X$ is a quasiaffinity (sf. [G16, Lemma 2.1 and Proposition 2.4]). Taking into account (2.7), we obtain that $T \sim S$. Therefore, $T$ is cyclic, and, by [M] or [K16, Proposition 16], $T$ is a.c..

Put

$$
\mathcal{M}=\operatorname{clos} X \omega_{a} H^{2} .
$$

By Theorem 2.4, T| $\left.\right|_{\mathcal{M}} \approx S$, and $Y \mathcal{M}=\omega_{a} H^{2}$ (because $g_{\alpha}$ is outer). Put

$$
T_{0}=\left.P_{\mathcal{M}^{\perp}} T\right|_{\mathcal{M}^{\perp}} \text {. }
$$

Then $T_{0}$ is cyclic, because $T$ is cyclic (see (1.1)), and $T_{0}$ is a.c., because $T$ is a.c. ([M] or [K16, Propositions 14 and 35]). It is easy to see that $\omega_{a}\left(T_{0}\right)=\mathbb{O}$. Indeed, denote by $\mathcal{H}$ the space in which $T$ acts. We have

$$
\mathcal{M}=\operatorname{clos} X \omega_{a} H^{2}=\operatorname{clos} X \omega_{a}(S) H^{2}=\operatorname{clos} \omega_{a}(T) X H^{2}=\operatorname{clos} \omega_{a}(T) \mathcal{H} .
$$

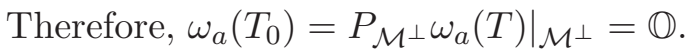

Finally, $T_{0}$ is not similar to a contraction by [C, Corollary 4.2]. Indeed, if $T_{0}$ is similar to a contraction, we can apply [C, Corollary 4.2] to $T$, because $\left.T\right|_{\mathcal{M}}$ is similar to an isometry, and $T$ is power bounded. Then $T$ must be similar to a contraction, a contradiction.

\section{REFERENCES}

[B] H. Bercovici, Operator theory and arithmetic in $H^{\infty}$, Math. Surveys Monogr., Amer. Math. Soc., v. 26, 1988.

[BP] H. Bercovici and B. Prunaru, Quasiaffine transforms of polynomially bounded operators, Arch. Math. (Basel), 71 (1998), 384-387.

[C] G. Cassier, Generalized Toeplitz operators, restriction to invariant subspaces and similarity problems, J. Oper. Theory, 53 (2005), no. 1, 4989. 
[DP] K. R. Davidson and V. I. Paulsen, Polynomially bounded operators, J. reine angew. Math., 487 (1997), 153-170.

[F] S. H. Ferguson, Polynomially bounded operators and Ext groups, Proc. Amer. Math. Soc., 124 (1996), 2779-2785.

[G03] M. F. Gamal', C.0-contractions: a Jordan model and lattices of invariant subspaces, Algebra i Analiz, 15 (2003), no. 5, 198-227 (in Russian); English transl.: St. Petersburg Math. J., 15 (2003), no. 5, 773-793.

[G12] M. F. Gamal', On the shift index of contractions, Acta Sci. Math. (Szeged), 78 (2012), 279-290.

[G16] M. F. Gamal', Examples of cyclic polynomially bounded operators that are not similar to contractions, Acta Sci. Math. (Szeged), 82 (2016), 597-628.

[G17] M. F. Gamal', A sufficient condition for the similarity of a polynomially bounded operator to a contraction, Zap. Nauchn. Sem. S.Peterburg. Otdel. Mat. Inst. Steklov. (POMI), 456 (2017), 77-95 (in Russian); English transl.: arXiv:1803.10174

[H] P. R. Halmos, Ten problems in Hilbert space, Bull. Am. Math. Soc., 76 (1970), 887-933.

[K89] L. Kérchy, Isometric asymptotes of power bounded operators, Indiana Univ. Math. J., 38 (1989), 173-188.

[K07] L. Kérchy, Shift-type invariant subspaces of contractions, J. Funct. Anal., 246 (2007), 281-301.

[K16] L. Kérchy, Quasianalytic polynomially bounded operators, in: Operator Theory: the State of the Art, Theta, Bucharest, 2016, 75-101.

[KS] L. Kérchy and A. Szalai, Asymptotically cyclic quasianalytic contractions, Studia Math., 223 (2014), 53-75.

[M] W. Mlak, Algebraic polynomially bounded operators, Ann. Polon. Math., 29 (1974), 133-139.

[N] N. K. Nikolski, Operators, functions, and systems: an easy reading. Volume I: Hardy, Hankel, and Toeplitz, Volume II: Model operators and systems, Math. Surveys and Monographs 92, AMS, 2002.

[Pa] V. I. Paulsen, Every completely polynomially bounded operator is similar to a contraction, J. Funct. Anal., 55 (1984), 1-17.

[Pi] G. Pisier, A polynomially bounded operator on Hilbert space which is not similar to a contraction, J. Amer. Math. Soc., 10 (1997), no. 2, 351-369.

[S] B. Sz.-Nagy, Diagonalization of matrices over $H^{\infty}$, Acta Sci. Math. (Szeged), 38 (1976), 223-238.

[SFBK] B. Sz.-Nagy, C. Foias, H. Bercovici and L. Kérchy, Harmonic analysis of operators on Hilbert space, Springer, New York, 2010. 
[T] K. Takahashi, On contractions without disjoint invariant subspaces, Proc. Amer. Math. Soc., 110 (1990), 935-937.

St. Petersburg Branch, V. A. Steklov Institute of Mathematics, Russian Academy of Sciences, Fontanka 27, St. Petersburg, 191023, Russia

E-mail address: gamal@pdmi.ras.ru 